\title{
Smoking cessation support for pregnant women: role of mobile technology
}

\author{
This article was published in the following Dove Press journal: \\ Substance Abuse and Rehabilitation \\ 12 April 2016 \\ Number of times this article has been viewed
}

\section{Christina L Heminger Jennifer M Schindler- \\ Ruwisch \\ Lorien C Abroms}

Department of Prevention and Community Health, Milken Institute School of Public Health, The George Washington University, Washington, DC, USA
Correspondence: Christina L Heminger Department of Prevention and Community Health, Milken Institute School of Public Health, The George Washington University, $950 \mathrm{New}$ Hampshire Avenue Northwest, Third Floor, Washington, DC 20052, USA

Tel +l 2029940146

Fax +I 2029943773

Email clh@gwu.edu
Background: Smoking during pregnancy has deleterious health effects for the fetus and mother. Given the high risks associated with smoking in pregnancy, smoking cessation programs that are designed specifically for pregnant smokers are needed. This paper summarizes the current landscape of mHealth cessation programs aimed at pregnant smokers and where available reviews evidence to support their use.

Methods: A search strategy was conducted in June-August 2015 to identify mHealth programs with at least one component or activity that was explicitly directed at smoking cessation assistance for pregnant women. The search for text messaging programs and applications included keyword searches within public health and medical databases of peer-reviewed literature, Google Play/iTunes stores, and gray literature via Google.

Results: Five unique short message service programs and two mobile applications were identified and reviewed. Little evidence was identified to support their use. Common tools and features identified included the ability to set your quit date, ability to track smoking status, ability to get help during cravings, referral to quitline, and tailored content for the individual participant. The theoretical approach utilized was varied, and approximately half of the programs included pregnancy-related content, in addition to cessation content. With one exception, the mHealth programs identified were found to have low enrollment.

Conclusion: Globally, there are a handful of applications and text-based mHealth programs available for pregnant smokers. Future studies are needed that examine the efficacy of such programs, as well as strategies to best promote enrollment.

Keywords: mHealth, smoking cessation, pregnancy, text messaging, mobile applications

\section{Introduction}

According to the US Pregnancy Risk Assessment Monitoring System, $\sim 10.7 \%$ of women smoked cigarettes during their pregnancy in $2010 .{ }^{1}$ In 2010, slightly more than half of smoking women $(54.3 \%)$ were able to quit while pregnant, which represents an increase from $43.2 \%$ in $2000 .{ }^{1}$ Additionally, postpartum rates of relapse among those who quit during pregnancy are high, with $\sim 40 \%$ relapsing. ${ }^{2}$ Certain populations, including young adults (20-24 years) (19.3\%) compared to women $35+$ years $(7.1 \%)$, those with $<12$ years of education $(22.5 \%)$ compared to those with higher educational attainments (6.5\%), those publically insured by Medicaid, the US social insurance program for low-income individuals and families (22.1\%), and those uninsured $(13.5 \%)$ compared to those otherwise insured (6.6\%), and American Indian (30.4\%)/Alaska Natives (21.1\%) compared to Hispanics (3.9\%), non-Hispanic Blacks (10.3\%), and non-Hispanic Whites $(15.9 \%)$ have the highest rates of smoking throughout pregnancy 
and after delivery. ${ }^{1}$ Overall, women who continue to smoke during pregnancy are more likely to have a smoking partner, lower socioeconomic status, fewer years of education, public insurance, high stress levels, less social support, increased addiction (younger initiation, more cigarettes daily), and unplanned pregnancy. ${ }^{3-5}$ Moreover, pregnant smokers may be hesitant to admit their smoking status to a provider or a public health practitioner, so the aforementioned smoking rates may be significantly underestimated. Self-reported measures are subject to social desirability bias and result in significant underreporting of cigarette smoking and consumption - underestimating true smoking prevalence by up to $25 \%$ from biochemically validated measures. ${ }^{6}$ This evidence has led to the best practice of requesting biochemical (ie, cotinine confirmed) measures to validate self-reported behavior. $^{7-10}$

A systematic review of the effects of cigarette smoking during pregnancy on child outcomes concluded that there are significant impacts of smoking on birth defects, including heart, limb, facial, dermatological, ocular, gastrointestinal, respiratory, genital, and musculoskeletal abnormalities. ${ }^{11}$ Additionally, smoking during pregnancy is associated with miscarriage, deprived fetal oxygen, placental bleeding, low birth weight, and premature delivery, as well as other more potentially long-term behavioral and cognitive effects. ${ }^{2,4}$ Women who smoke after delivery increase their child's risk of sudden infant death syndrome (SIDS), asthma, and ear infection. ${ }^{2}$ The mother is also at risk for a myriad of smoking-related morbidities, including cancers, cardiovascular diseases, and pulmonary diseases. ${ }^{2,12}$ Therefore, the health benefits of quitting during pregnancy and remaining abstinent after birth are enormous for both the mother and the baby. To this end and in response to failed target goals for Healthy People 2010, Healthy People 2020 has specified several objectives related to abstinence during pregnancy, including a $10 \%$ increase in abstinence among pregnant smokers and a decrease in relapse after delivery among those who successfully quit during pregnancy. ${ }^{1,13}$

According to the Clinical Practice Guidelines issued by the US Public Health Service, a broad range of cessation approaches are recommended for pregnant smokers, including assessment of smoking status, counseling directly from a provider, and referral to a cessation-specific counselor or quitline that includes skill-based training and social support. ${ }^{14}$ However, as noted in these guidelines, not all general cessation recommendations (ie, nicotine replacement therapy [NRT]) are recognized as safe and/ or appropriate for pregnant women. ${ }^{14}$ One study using the
HealthStyles (C) 2008 survey suggests that despite varying levels of knowledge about the health effects of smoking on the fetus throughout pregnancy, most smokers of reproductive age still express willingness to quit during pregnancy, ${ }^{15}$ indicating the importance of providing evidence-based approaches for cessation in this population. Despite motivation to quit, pregnant smokers may face additional disadvantages that challenge the effectiveness of traditional cessation methods. Pregnancy inherently creates a competing priority against smoking cessation, and historically, attendance in smoking cessation programs for pregnant women has been low. ${ }^{16}$

Not surprisingly, pregnant women tend to underutilize evidence-based cessation practices such as counseling or the use of quitlines. ${ }^{17}$ Given the stigma associated with smoking during pregnancy and the difficulty in providing evidence-based counseling and other behavioral interventions to this population, the emerging field of mobile health (mHealth) may be an underexplored avenue to better support these tailored and targeted interventions. mHealth interventions have several notable advantages to typical provider-based interventions, including reach and accessibility to a wider range of women in child-bearing age at low or no cost, increased privacy for participants, and tools and resources at participants' fingertips beyond a clinical visit.

In the USA, $85 \%$ of all adults have mobile phones, and $72 \%$ of mobile phone owners send and receive text messages. ${ }^{18}$ Additionally, $64 \%$ of Americans are smartphone owners, allowing them to text message as well as use mobile applications (apps); this is a marked increase from 35\% owning smartphones in $2011 .{ }^{19}$ Smartphones provide telephonic and internet access to three groups most readily: younger (ages 18-29 years), low socioeconomic status, and non-White adults. ${ }^{19}$ Short message service (SMS) - or text messaging interventions at large have generally had positive impacts on cessation among adult smokers. ${ }^{20-22}$ However, to date, little has been done to evaluate text-based interventions to determine their efficacy specifically among pregnant smokers or to look specifically at mobile apps available for this population. While many text messaging interventions in this population have been piloted, to date, none have yet been implemented with adequate sample size or control group. A broad review of apps on a variety of health behaviors not only indicated their applicability to addiction and cessation behaviors but also demonstrated the inherent challenges to rapidly changing and available technologies in terms of properly vetting and understanding the implications of these tools. ${ }^{23} \mathrm{~A}$ content analysis 
of apps specifically for cessation not only found strengths of several of these mHealth interventions but also identified areas for improvement to help these technologies better meet guidelines and current cessation recommendations. ${ }^{14,24}$ While several apps have been developed specifically for pregnant women attempting to quit smoking, no apps tailored to this population have currently been evaluated.

Given the high risks associated with smoking in pregnancy and the widespread use of mobile phones in this population, smoking cessation programs that are designed specifically for pregnant smokers on mobile phones may be promising. The purpose of this paper is to summarize mHealth programs (text messaging and app-based interventions) currently available for pregnant smokers and, where available, to summarize study results and outcomes.

\section{Methods}

\section{Search strategy}

A search was conducted to identify mobile phone-based programs with at least one component or activity that was explicitly directed at smoking cessation assistance for pregnant women. In order to be included in this review, a program must 1) include a mHealth element (text messaging or mobile app) explicitly designed to promote smoking cessation among women during and/or after pregnancy and 2) report on at least one smoking cessation outcome. Cessation programs aimed at a larger target audience (ie, "adults") but with an explicit pregnancy component (ie, topical listing or menu for pregnant women or ability to tailor results based on pregnant status) were also included. General text messaging programs and applications that made no mention of pregnancy were, therefore, not included. There were neither date nor language exclusions set.

The research team conducted a three-phase search in June 2015, the first of which was a search of the following peer-reviewed databases: PubMed, PsychINFO, and Medline. Search terms included combinations of "SMS", "text", "text messag*", "app", "mobile app", and "mobile application" paired with both "pregnant" and "smoking". This search yielded 69 results. Thirty-seven articles were duplicates found among the search terms, 14 articles were review articles lacking original data, and 12 articles described programs that did not contain a mHealth component or did not explicitly address the target population of pregnant smokers. The six $(8.6 \%)$ remaining peer-reviewed articles described four unique text messaging programs. These are discussed in Table 2.

Next, the research team conducted a scan of the iTunes and GooglePlay app stores to identify mobile apps related to pregnancy and smoking cessation. Search terms included combinations of "SMS", "text", "text messag*", "app", "mobile app", and "mobile application" paired with both "pregnant" and "smoking". This search yielded 86 results. After removing non-English applications and those outside the scope of addressing smoking cessation during pregnancy, only two applications remained: Quit for You - Quit for Two and SmokeFree Baby. The number of downloads for each application was recorded from GooglePlay; download ranges within GooglePlay (ie, "100-500 downloads") only represent users utilizing the Android platform. The iTunes store does not provide download information; therefore, these totals do not account for iPhone users and likely underrepresent the total number of app downloads. An additional search was conducted for evaluation data for these specific applications in the aforementioned databases and within Google gray literature; no data were found as of June 2015.

Finally, the research team performed gray literature searches using Google; as mentioned earlier, search terms included combinations of "SMS", "text", "text messag*", "app", "mobile app", and "mobile application" paired with both "pregnant" and "smoking". The top 20 search results for each search term combination were evaluated for uniqueness and relevance. This search yielded one unique program that met the inclusion criteria and had not yet been discovered through previous search strategies: the SmokefreeMOM text messaging program. As of August 2015, no evaluation data were available for this program.

In addition, in August 2015, inquiries were made to the aforementioned study authors, app developers, and the SmokefreeMOM program contacts to inquire whether any evaluation data (preliminary data, drafted manuscripts, or conference presentations) were available to supplement the publically available data analyzed for this review; no outcome data were available at the time of publication. However, where available, we obtained subscriber/enrollment data; this information is captured in Table 2.

\section{Article coding}

Each article was reviewed and scanned for data by one member of the research team using an a priori coding scheme adapted from the meta-analysis of text messaging interventions by Head et al. ${ }^{20}$ For each variable, relevant text was extracted from the published articles, and together two team members discussed and decided on each appropriate code. Modifications to the codebook were made after the analysis was complete to streamline the variable categories; all three 
authors determined the final coding scheme. The final codes included study characteristics such as year and journal published, program country and sponsor, sample size/enrollment, and study design and program features such as intervention type (SMS/text messaging or app), content (focused solely on smoking cessation during pregnancy or additional elements related to general pregnancy health), program tools and features, referral mechanisms, interaction with participants, theoretical basis, message tailoring, and message dose. The coders met after all programs were coded to discuss the results and potential revisions to the codebook. Definitions for each variable can be found in Table 1 .

For the three programs for which no peer-reviewed literature was currently available (SmokefreeMOM; Quit for You - Quit for Two; SmokeFree Baby), theoretical mechanism and outcomes could not be ascertained and are, therefore, not indicated in the results. Only information included in peer-review publications and gray literature or shared in writing with the research team was included in this review; assumptions and extrapolations were not made.

\section{Results}

In total, seven unique mHealth programs promoting smoking cessation among pregnant women were included in this analysis. A review of all programs meeting the inclusion criteria $(\mathrm{N}=7)$ can be found in Table 3. These include five unique SMS programs - Quit4Baby, MiQuit, SMAT, SmokefreeMOM, and one that was unnamed, but we refer to it by the following acronym, SGR, representing the approach of using scheduled gradual reduction (SGR) - and two mobile apps - Quit for You - Quit for Two, and SmokeFree Baby. The peer-reviewed search highlighted Quit4Baby, ${ }^{25}$ MiQuit, ${ }^{26-28}$ SMAT, ${ }^{29}$ and SGR $;{ }^{30}$ no apps were mentioned in the published literature.

The studies were located in the United States (Quit4Baby, SGR, SmokefreeMOM), the United Kingdom (MiQuit, SmokeFree Baby), Australia (Quit for You - Quit for Two), and Canada (SMAT), were sponsored by national health agencies, and ranged in enrollment from 20 to 50,000 participants. Table 2 shows the additional study characteristics.

Table I Coding scheme definitions

\begin{tabular}{|c|c|}
\hline Category & Definition \\
\hline \multicolumn{2}{|l|}{ Intervention } \\
\hline SMS/text messaging & A text message/SMS program \\
\hline App & A downloaded mobile application \\
\hline \multicolumn{2}{|l|}{ Content } \\
\hline Smoking cessation & Content related to quitting smoking or information about smoking \\
\hline General pregnancy & Content related to prenatal health or pregnancy tips \\
\hline \multicolumn{2}{|l|}{ Tools/features } \\
\hline Ability to set quit date & Does the program allow the participant to proactively set a quit date either at onset or during program? \\
\hline Smoking tracker & Does the program include regular monitoring of smoking status? \\
\hline Cravings help & Can the participant ask for help during a craving by typing a keyword or using a tool within the program? \\
\hline Smoked help & Can the participant inform the program they smoked a cigarette and receive advice/tips? \\
\hline Calculator & Can the participant calculate monetary savings or other smoking-related milestones within the program? \\
\hline Games or distractions & Can the participant play games or engage in a distraction tool? \\
\hline Linked media & Are there links to or embedded videos or pictures in the program? \\
\hline \multicolumn{2}{|l|}{ Referrals } \\
\hline Quitline & Does the program provide a direct referral to the quitline number? \\
\hline NRT & Does the program recommend or discuss NRT for pregnant women? \\
\hline \multicolumn{2}{|l|}{ Implementation features } \\
\hline \multicolumn{2}{|l|}{ Interaction } \\
\hline One-way messages & Only the program can send the participant messages \\
\hline Two-way messages & The program can send participant messages and the participant can send messages to the program \\
\hline Live help available & The program has a live contact that can talk with participants or answer questions \\
\hline Theoretical basis/approach & Do the articles explicitly state that they used a behavioral theory or approach? \\
\hline Tailored messages & $\begin{array}{l}\text { Are the messages tailored to/around the participant's quit date and due date or do they utilize personalized } \\
\text { content such as the participant's name or their motivators for quitting? }\end{array}$ \\
\hline \multicolumn{2}{|r|}{ 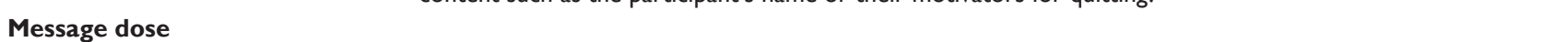 } \\
\hline Fixed & Messages come at a prescribed frequency throughout the program (ie, one message per day) \\
\hline Decreasing & Message frequency tapers over time throughout the program \\
\hline Individualized & Message frequency depends on individual characteristics such as quit date, due date, or participant preferences \\
\hline Varied & Message frequency differs from day to day throughout the program \\
\hline
\end{tabular}

Abbreviations: SMS, short message service; NRT, nicotine replacement therapy. 


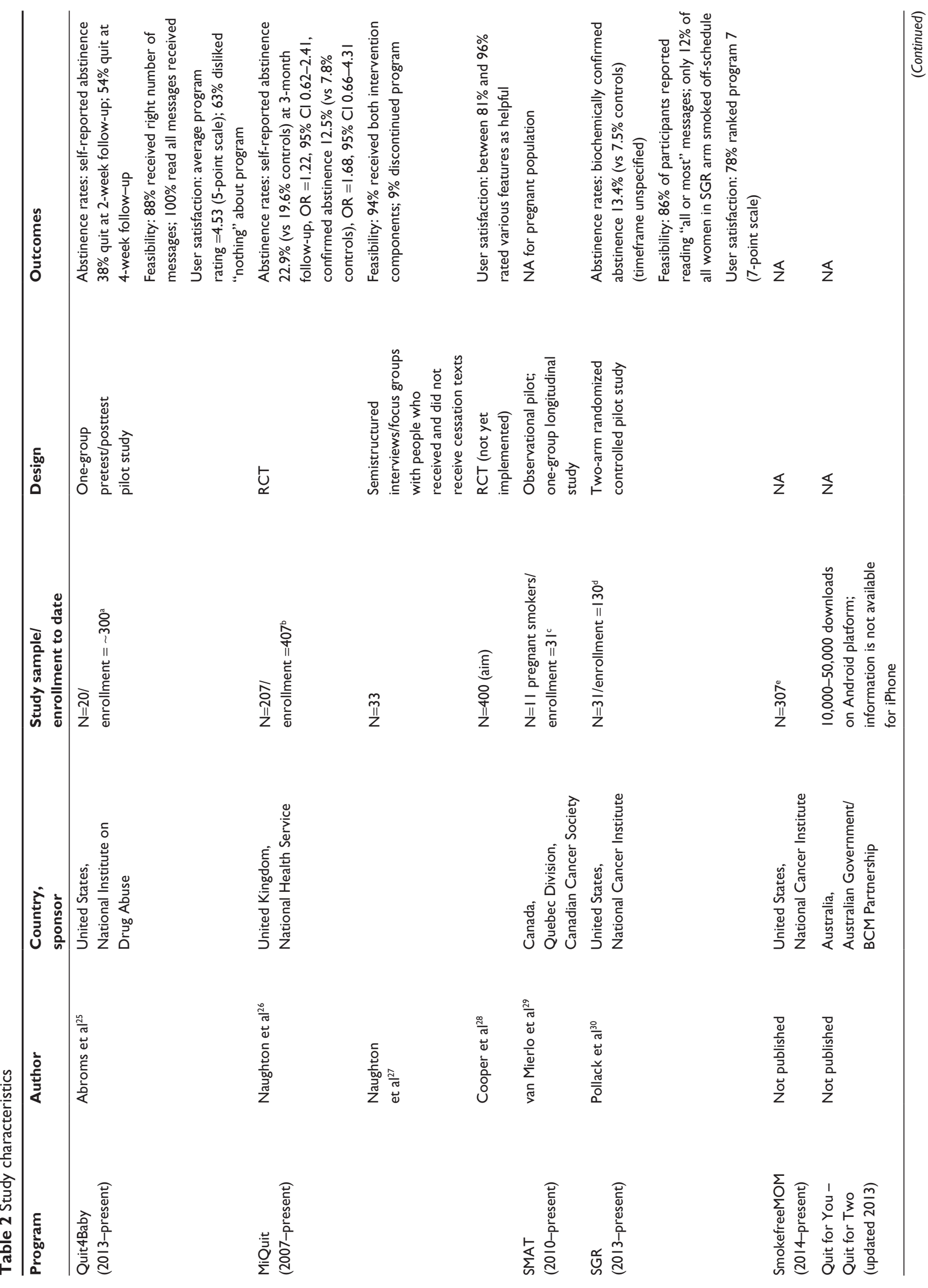




\section{Program overviews Quit4Baby}

Quit4Baby is a text-based cessation intervention for pregnant smokers that was adapted from Text2quit, an evidenced-based program for the general smoking population. ${ }^{25}$ It strives to provide smoking cessation and general pregnancy information to expectant mothers. Keywords include "date", "track", "crave", "tips", "game", "smoked", and "slip". Participants are provided referrals to the quitline by texting "tips". The frequency of texts varies based on the quitting protocol: prequit, quit date, postquit, and ultimately the participant's due date, with the highest frequency of texts around the participant's self-determined quit date.

\section{MiQuit}

MiQuit is a text-based self-help intervention that delivers tailored smoking cessation support by text message and four-page paper leaflet to pregnant smokers. ${ }^{26-28}$ Keywords include "help" for struggling moments and "slip" for a lapse or recurrence of smoking. It does not provide referrals to a quitline. The leaflet is highly tailored for each participant based on 20 characteristics provided in the baseline survey, including but not limited to participant's name, beliefs about harms of prenatal smoking, motivations for quitting, confidence in quitting ability, nicotine dependence, situational smoking and temptations, and presence of other household smokers. Texts are separated by quit status protocol (prequit, postquit), which is assessed twice during the 12-week study. Text frequency is highest during the first 4 weeks and then reduces with participants receiving zero, one, or two texts per day and having the ability to change this frequency by texting "more" or "less".

\section{SMAT}

SMAT is a text-based cessation intervention to help young adult Quebecers quit smoking. ${ }^{29}$ This program offers text messages plus the option for real-time chat sessions with counselors, referred to as the Text and Chat Integrated design. While it is unclear how the pregnancy algorithm differs from the general smoking population content, it is clear that participants receive messages related to cravings, opportunities to report a slip, and referral to a quitline for support. Keywords include "edgy", "alcohol”, "stress", "slip", “craving", "smokers", and "motivate". The message frequency varies from one message per day to two messages per day on the participant's quit date and in weeks 5-12 of the 12-week program.

\section{SGR}

SGR is a text-based cessation intervention for pregnant smokers, which delivers support and a SGR plan to promote cessation 
Table 3 Program characteristics

\begin{tabular}{|c|c|c|c|c|c|c|c|c|}
\hline Category & $\begin{array}{l}\text { Quit4Baby } \\
(\text { Abroms } \\
\text { et } \mathrm{al}^{25} \text { ) }\end{array}$ & $\begin{array}{l}\text { MiQuit } \\
\text { (Naughton } \\
\text { et } \mathrm{al}^{26-28} \text { ) }\end{array}$ & $\begin{array}{l}\text { SMAT } \\
\text { (van Mierlo } \\
\text { et } \mathrm{al}^{29} \text { ) }\end{array}$ & $\begin{array}{l}\text { SGR } \\
(\text { Pollack } \\
\left.\text { et } \mathrm{al}^{30}\right)\end{array}$ & $\begin{array}{l}\text { Smokefree } \\
\text { MOM }\end{array}$ & $\begin{array}{l}\text { Quit for } \\
\text { You - Quit } \\
\text { for Two }\end{array}$ & $\begin{array}{l}\text { SmokeFree } \\
\text { Baby }\end{array}$ & Total \\
\hline \multicolumn{9}{|l|}{ Intervention } \\
\hline SMS/Text & $\mathrm{x}$ & $x$ & $x$ & $x$ & $x$ & & & 5 \\
\hline App & & & & & & $x$ & $x$ & 2 \\
\hline \multicolumn{9}{|l|}{ Content } \\
\hline Smoking cessation & $x$ & $x$ & $x$ & $x$ & $x$ & $x$ & $x$ & 7 \\
\hline General pregnancy & $x$ & & & & $x$ & $x$ & $x$ & 4 \\
\hline \multicolumn{9}{|l|}{ Tools/features } \\
\hline Ability to set quit date & $x$ & $x$ & $x$ & $x$ & $x$ & $x$ & $x$ & 7 \\
\hline Smoking tracker & $x$ & $x$ & & $x$ & $x$ & & $x$ & 5 \\
\hline Cravings help & $x$ & $x$ & $x$ & & $x$ & $x$ & $x$ & 6 \\
\hline Smoked help & $x$ & $x$ & $x$ & & $x$ & & & 4 \\
\hline Calculator & $x$ & & & & & $x$ & $x$ & 3 \\
\hline Games or distractions & $x$ & $x$ & $x$ & & $x$ & $x$ & $x$ & 6 \\
\hline Linked media & $x$ & & & & $x$ & & $x$ & 3 \\
\hline \multicolumn{9}{|l|}{ Referrals } \\
\hline Quitline & $x$ & & $x$ & & $x$ & $x$ & $x$ & 5 \\
\hline NRT & & & & & & & $x$ & 1 \\
\hline \multicolumn{9}{|l|}{ Implementation features } \\
\hline Two-way interaction & $x$ & $x$ & $x$ & $x$ & $x$ & & & 5 \\
\hline Live help available & & & $x$ & & $x$ & & & 2 \\
\hline $\begin{array}{l}\text { Theoretical basis/approach } \\
\text { cited }\end{array}$ & $x$ & $x$ & $x$ & $x$ & & NA & NA & 4 \\
\hline Tailored messages & $x$ & $x$ & $x$ & $x$ & $x$ & $x$ & $x$ & 7 \\
\hline Message dose & & & & & & NA & NA & \\
\hline Decreasing & $x$ & $x$ & $x$ & $x$ & $x$ & & & 5 \\
\hline Individualized & $x$ & $x$ & $x$ & $x$ & $x$ & & & 5 \\
\hline Varied & & & $x$ & & & & & 1 \\
\hline Total & 16 & 12 & 14 & 9 & 15 & 9 & 12 & \\
\hline
\end{tabular}

Abbreviations: SGR, scheduled gradual reduction; SMS, short message service; NRT, nicotine replacement therapy; NA, not applicable.

during pregnancy. ${ }^{30}$ While the program does not appear to provide referrals to quitlines or counselors, study staff call to check in with participants to assess adherence to the SGR plan. Each week, participants receive a newly themed support text including but not limited to reasons for quitting, preparing to quit, partner smoking status, and handling slips. Participants are asked to select a quit date 2-3 weeks from the onset of the program. The SGR group receives the supportive text messages plus alert texts prompting them to smoke on a regimented schedule. Participants receive a varied amount of texts, approximately one to three, but upward of five per day. The program lasts for 5 weeks.

\section{SmokefreeMOM}

SmokefreeMOM is a text-based cessation intervention for pregnant smokers based on SmokefreeTXT but tailored to this population. SmokefreeMOM is described on women. smokefree.gov as a free text messaging service "that provides 24/7 tips, advice, and encouragement to help pregnant women quit smoking". The website further describes the program to be tailored around the woman's quit date and due date as well as to allow her to select one of three options: ready to quit, wanting to cut down, and not yet ready to quit but would like to receive motivational messaging. There are also BabyTips on general pregnancy tips and advice.

\section{Quit for You - Quit for Two}

This is an app developed for Android and iPhone users that has options to play baby-related distraction games or learn about quitting.

\section{SmokeFree Baby}

This is an app developed for Android and iPhone users that includes routine status check-ins, tools, stress-relieving options, health effects, a personalized profile, and more.

\section{Program characteristics}

All five SMS programs instructed and reminded participants to set a quit date; SGR had their SMS-only group select a quit 
date and their SMS + SGR group would just receive tapering messages in line with SGR. Four SMS programs enlisted routine smoking status-tracking mechanisms and assistance with cravings or smoking relapses and report having games and other distractions. Quit4Baby also included a calculator to demonstrate cost savings from quitting smoking, and both Quit4Baby and SmokefreeMOM included multimedia content such as links to or embedded videos or photos. The apps also included an option to set a quit date, cravings help, calculators, and games. SmokeFree Baby also included routine tracking of smoking and multimedia options such as the ability to record voice memos or reminders and watch informational videos.

Quit4Baby, SMAT, and SmokefreeMOM, in addition to the two apps, referred participants to a national quitline to assist with smoking cessation outside the SMS program. None of the programs explicitly mentioned detailed information about NRT beyond advising the participant to talk with their provider, with the exception of the SmokeFree Baby app.

All five SMS programs personalized messages for their individual participants - utilizing names, personal information, or tapping into unique participant characteristics such as quit date and due date - and engaged participants with two-way interaction, allowing participants to receive texts from the program and to send keywords or text messages to the program. Four of the five SMS programs (excluding SMAT) had automated text messages sent to participants on a time schedule and/or in response to a participant-generated keyword (ie, an automated response to a participant texting "CRAVE" when experiencing a smoking craving). SMAT and SmokefreeMOM were the only programs that offered live assistance to participants, and SmokefreeMOM users could only do this from the women.smokefree.gov webpage. SMAT actually allowed users to "text chat" with a counselor through their Text and Chat Integrated component. SMAT also offered a varied text messaging schedule, while the remaining programs followed similar patterns of message frequency decreasing over time in relation to a participant's individualized quit date.

Behavioral theory has been effectively utilized in the smoking cessation arena, most notably the Social Cognitive Theory ${ }^{31}$ and the Health Belief ${ }^{32}$ and Transtheoretical ${ }^{33}$ Models. ${ }^{34}$ Three of the five SMS programs explicitly stated that they were based on behavioral theory: both Quit4Baby and MiQuit utilized Social Cognitive Theory. Additionally, MiQuit based their program on the Perspectives on Change ${ }^{35}$ and the Elaboration Likelihood Model. ${ }^{36}$ SMAT was based on the Health Belief Model, Transtheoretical Model, and motivational interviewing techniques. Additionally, as its name implies, SGR utilizes a systematic SGR of cigarettes smoked, which is also considered to be a guiding approach. We did not have sufficient information from the nonpublished programs (SmokefreeMOM; Quit for You - Quit for Two; and SmokeFree Baby) to determine their theoretical underpinnings.

Three of the five SMS programs reported limited abstinence, feasibility, and satisfaction-related outcomes from their pilot studies. Of these, only two studies employed the gold standard of collecting biochemically confirmed quitting validation (MiQuit and SGR). Quit4Baby $(\mathrm{N}=20)$ demonstrated promising self-reported abstinence rates, with $38 \%$ of participants having quit at the 2 -week follow-up and $54 \%$ having quit at the 4 -week follow-up. The majority $(88 \%)$ of the participants felt they received the "right number" of messages, that the messages came at the right time of day, and $100 \%$ of respondents reported reading all messages received. Users rated the program favorably, giving an average rating of 4.53 out of a maximum of 5 points. MiQuit $(\mathrm{N}=207)$ also reported higher self-reported abstinence rates for the intervention group (22.9\% compared to $19.6 \%$ among controls) at the 3-month follow-up and an odds ratio of 1.22 but failed to achieve statistical significance $(95 \%$ confidence interval 0.62-2.41). Similarly, biochemically confirmed abstinence rates ( $12.5 \%$ compared to $7.8 \%$ among controls) were higher for the intervention participants but not statistically significant $(95 \%$ confidence interval $0.66-4.31)$. The vast majority of participants received both intervention components (SMS program and tailored leaflet) and only 9\% discontinued text messaging at any point in the study. A range of $81 \%-96 \%$ of users rated each program feature as "helpful". SGR $(\mathrm{N}=31)$ reported higher biochemically confirmed abstinence rates for SGR participants (13.4\%) compared to control participants (7.5\%). Eighty-six percent of the participants reported reading "all or most" of the text messages, and of the participants enrolled in SGR, only $12 \%$ smoked outside of their prescribed schedule. More than three quarters of participants (78\%) gave the program a satisfaction score of 7 out of a maximum of 7 points. Results for pregnant smokers enrolled in SMAT were not made available, and SmokefreeMOM and the mobile apps did not have published data available.

Table 3 contains the complete list of program features analyzed.

\section{Mobile application characteristics}

It is worth briefly mentioning that the two apps have different content and design features, although we cannot speak 
directly to their effectiveness yet. First, Quit for You - Quit for Two is designed with bright colors and cartoon characters to give a vibrant and playful feel. The two main tabs include "Play" and "Learn" as well as a visible link to the Quitline and a Profile that highlights on the first page approximate savings in dollar amount, cigarettes not smoked, and days since you quit. The Play tab details ways to distract oneself during cravings such as baby games, baby names, and breathing strategies. The Learn tab highlights quit tips, fetal growth milestones, and equating savings with items you can now "buy". In comparison, SmokeFree Baby is designed as dual-toned, with a more serious character for the design. The home screen welcomes the user back to the program by name and asks how they are doing as well as a yes/no response for whether they smoked yesterday, followed by feedback. Once in this main tab, they are offered six boxes for easy navigation to the toolbox (tips, inputting supportive contact numbers, education on NRT, and video memos), identity (creating profile and customizing app), and stress relief, as well as content related to health education, referrals, and behavioral modification. They can also navigate to FAQs, About (team, program, and contact information), Settings (notifications and start date), and Tools to Quit (including addiction information, reasons to quit, getting ready to quit, and withdrawal information). The top of the home screen also lists the number of smoke-free days and number of smoke-free days in a row, along with customization by name.

\section{Discussion}

The objective of this review was to assess the landscape of mobile health interventions promoting smoking cessation for pregnant women, a particularly vulnerable population. mHealth interventions offer a unique opportunity to provide tailored, convenient, and private support to an at-risk population, and the seven programs extracted in this review show promise in meeting their needs.

The vast majority of pregnant smokers in the USA have mobile phones, and a significant majority has smartphones. ${ }^{18}$ Despite the nearly ubiquitous use of mobile phones in this population, very few programs and apps were identified ( $\mathrm{N}=5$ text programs, $\mathrm{N}=2$ apps). This is far fewer than the number of programs available for the adult smoking population which number in the hundreds for apps alone. ${ }^{37}$ Given that commercial organizations do not seem to be producing applications for pregnant smokers, government-funded initiatives may be a needed service for this population. From a global perspective, the seven programs identified are all running in English-speaking developed nations (USA, UK, Canada, and
Australia). There is a need for mHealth programs that address pregnant smokers globally, particularly those living in countries with the highest smoking rates and those with limited access to prenatal care or smoking cessation support.

Few studies have been conducted to evaluate mHealth programs in this population, and the majority of these studies have consisted of pilots. This review not only described what was available but also confirmed serious methodological deficits in this area of treatment and research. While three of the four published SMS programs collected some evaluation outcomes related to participant abstinence, satisfaction, and program feasibility, data were limited and comparisons between programs are currently impossible given the wide variety of metrics utilized. Additionally, there is not currently any published literature on the feasibility, acceptability, or effectiveness of either of the two available mobile apps for pregnant smokers. Of the published SMS interventions, most were pilot tests, and small sample sizes limited the opportunity for rigorous statistical analysis of program effectiveness. However, when speaking with the program authors and searching Clinicaltrials.gov, it is apparent that randomized controlled trials and other efficacy trials are underway; therefore, additional outcome data may be forthcoming. Such trials should ensure collection of biochemically confirmed cessation measures, given the methodological concerns with relying on self-reported information. ${ }^{6}$ To date, no valid biochemically confirmed behavioral impact evaluation with adequate sample size of a mHealth treatment for pregnant smokers has been reported in the peer-review literature.

Generally, enrollment numbers were low among the textbased programs. The fact that most programs represent pilot programs may explain the relatively low enrollment numbers (20-130 participants). For example, SmokefreeMOM only currently reports 307 active subscribers. However, when looking at those women ever enrolled in the program, $>800$ have registered. While it is understood that the program is supposed to be temporal in that it will only be relevant for users who are trying to quit or currently pregnant, additional insight into unsubscription rates and reasons for unsubscribing will be of particular interest. Further, when examining the apps, SmokeFree Baby only has 100-500 installs, while Quit for You - Quit for Two has 10,000-50,000 installs (GooglePlay only until lists ranges, not exact numbers). Both apps are currently available from the app store, but one has grown by leaps and bounds, while the uptake of the other remains limited. This difference could be for many reasons, including the fact that SmokeFree Baby is currently undergoing testing and may not be offered widely but mainly 
distributed to test or pilot participants. Additionally, it could be the program developer or sponsor (ie, Australian government vs independent application) that promotes enrollment. Coordinated efforts are needed to reach and enroll pregnant smokers - this is a universal challenge among smoking cessation programs.

Interestingly, all the programs included functions to set quit dates, as well as keywords or tools that participants could use to solicit support, distractions, or information throughout their quitting process. Four of the seven included both smoking cessation content as well as pregnancy information and tips; the integration of both prenatal information and smoking cessation information provides a "one stop shop" for pregnant smokers and may help provide much needed information and resources to women without access to routine prenatal care or support networks. Most were theory-based focusing on the constructs of self-efficacy and attitudes to promote change and utilizing the stages of change to measure and track progress. The SGR program utilized a unique approach in SGR, which may be promising for pregnant women already faced with daunting physical and emotional changes during pregnancy. All SMS programs utilized two-way interaction, demonstrating the importance of an engaged and autonomous user, and most utilized an SMS schedule that decreased after a participant's quit date has passed, providing targeted help around a quit date and sustained support throughout the cessation process. Each and every text program and app tailored their content to the individual in some way - utilizing their first name, quit date, due date, or other imputed data such as supportive friends/family members' names, personalized reasons and motivation for quitting, and desired number and timing of messages received. This is not surprising given the data linking tailored messages to participant engagement and satisfaction. ${ }^{26}$ Only one program - the Smokefree Baby application, based in the UK - provided explicit information on NRT options and their feasibility for use during pregnancy. Currently, the US Clinical Preventive Guidelines do not have enough evidence to support the widespread use of NRT for pregnant women, but if/as evidence emerges to promote pharmacotherapy in this population, US-based text programs and apps should consider inclusion of this information.

\section{Strengths and limitations}

This study represents the first review of mobile cessation programs explicitly providing cessation support to pregnant women. A key strength of this study is the inclusive and comprehensive nature of the search strategy (systematic searches of peer-reviewed publications, gray literature [ie, Google searches for programs, scans of program websites], application stores, and personal communications with program developers), which ensured inclusion of both text-based programs and applications, programs with both published and unpublished data, and the most current enrollment and outcome data available.

That said, the results herein represent assertions gathered from the aforementioned sources, and it is possible that there are more than seven programs available that address pregnant smokers. Moreover, some of the programs described here might contain additional tools, features, or content not uncovered by the research team. For example, the study team was not able to determine how theoretical frameworks were utilized in a given program, just the assertion that a theory had been included in published literature. Another limitation includes limited reach data. The application download statistics shared here were collected from GooglePlay and, therefore, only represent downloads from Android users; iPhone download information is not publically available. It can be assumed that the download numbers for the two applications depicted here are underestimates, and it is possible that iPhone download figures might be comparable to those found in GooglePlay. While this study does not provide firm numbers on application reach, the subset of publically available data can still demonstrate usage and acceptability of mobile apps.

\section{Conclusion}

Text messaging programs for a variety of health behaviors, including smoking, have been previously evaluated, but among pregnant smokers specifically, there are a growing number of mHealth tools in both SMS and app format that may benefit this particularly hard to reach population. Given that SMS and mobile apps may be especially costintensive to develop and test, understanding the evidence base is crucial to ensure that new technologies incorporate previous findings or can specifically test modifications of current designs to avoid having a proliferation of tools that are untested and ineffective. The field could greatly benefit from a more systematic analysis of current programs to guide best practices. Pregnant women remain in urgent need and are primed for participation given prominent mobile and smartphone usage and the appeal of mHealth interventions for a stigmatized behavior. Therefore, the authors call for further research with urgency to ensure evidence-based mobile interventions targeting this critical health behavior change are made available to this vulnerable population as rapidly as results are available. 


\section{Disclosure}

The George Washington University/Dr Lorien C Abroms has licensed the Quit4Baby program to Voxiva, Inc.; Dr Lorien C Abroms has stock options in Voxiva, Inc. The authors report no other conflicts of interest in this work.

\section{References}

1. Tong VT, Dietz PM, Morrow B, et al. Trends in smoking before, during and after pregnancy - pregnancy risk assessment monitoring system, United States, 40 sites, 2000-2010. MMWR. 2013;62(6):1-19.

2. Center for Disease Control and Prevention [webpage on the Internet]. Tobacco Use and Pregnancy. [n.d.]. 2014. Available from: http://www. cdc.gov/reproductivehealth/tobaccousepregnancy/. Accessed July 7 , 2015.

3. McLeod D, Pullon S, Cookson T. Factors that influence changes in smoking behaviour during pregnancy. N Z Med J. 2003;116(1173):U418.

4. Murin S, Rafii R, Bilello K. Smoking and smoking cessation in pregnancy. Clin Chest Med. 2011;32(1):75-91.

5. Gilbert NL, Nelson CR, Greaves L. Smoking cessation during pregnancy and relapse after childbirth in Canada. J Obstet Gynaecol Can. 2015;37(1):32-39.

6. Stookey GK, Katz BP, Olson BL, Drook CA, Cohen SJ. Evaluation of biochemical validation measures in determining smoking status. J Dent Res. 1987;66(10):1597-1601.

7. Nanji AA, Lawrence AH. Skin surface sampling for nicotine: a rapid, noninvasive method for identifying smokers. Int $J$ Addict. 1998;23(11):1207-1210.

8. Luepker RV, Pallonen UE, Murray DM, Pirie PL. Validity of telephone surveys in assessing cigarette smoking in young adults. Am J Public Health. 1989;79(2):202-204.

9. Murray DM, O'Connell CM, Schmid LA, Perry CL. The validity of smoking self-reports by adolescents: a reexamination of the bogus pipeline procedure. Addict Behav. 1987;12(1):7-15.

10. Vesey CJ, Saloojee Y, Cole PV. Cigarette consumption and biochemical measures of smoke intake. Br Med J. 1982;285(6340):507-508.

11. Hackshaw A, Rodeck C, Boniface S. Maternal smoking in pregnancy and birth defects : a systmatic review based on 173,687 malformed cases and 11.7 million controls. Hum Reprod Update. 2011;17(5): 589-604.

12. U.S. Department of Health and Human Services; Centers for Disease Control and Prevention; National Center for Chronic Disease Prevention and Health Promotion; et al. [webpage on the Internet]. The Health Consequences of Smoking-50 Years of Progress: A Report of the Surgeon General. 2014. Available from: http://www.surgeongeneral.gov/library/ reports/50-years-of-progress/exec-summary.pdf. Accessed July 16 , 2015.

13. Office of Disease Prevention and Health Promotion [webpage on the Internet]. Healthy People 2020: Maternal, Infant, and Child Health. [n.d.]. 2015. Available from: http://www.healthypeople.gov/2020/ topics-objectives/topic/maternal-infant-and-child-health/objectives. Accessed August 10, 2015.

14. Fiore MC, Jaen CR, Baker TB, et al. Treating Tobacco Use and Dependence: 2008 Update. 2015. Available from: http://www.ahrq.gov/sites/ default/files/wysiwyg/professionals/clinicians-providers/guidelinesrecommendations/tobacco/clinicians/update/treating_tobacco_use08. pdf. Accessed June 23, 2015.

15. Polen KN, Sandhu PK, Honein MA, et al. Knowledge and attitudes of adults towards smoking in pregnancy: results from the HealthStyles $\subset$ 2008 survey. Matern Child Health J. 2015;19(1):144-154.

16. Borland R, Segan CJ. The potential of quitlines to increase smoking cessation. Drug and Alcohol Rev. 2006;25(1):73-78.
17. North American Quitline Consortium (NAQC). Quitline Services for Pregnant and Postpartum Women: A Literature and Practice Review. 2014. Available from: http://c.ymcdn.com/sites/naquitline.site-ym. com/resource/resmgr/Issue_Papers/PregnantPostpartumIssuePaper. pdf. Accessed February 17, 2016.

18. Pew Research Center [webpage on the Internet]. The Smartphone Difference. 2015. Available from: http://www.pewinternet.org/2015/04/01/ us-smartphone-use-in-2015. Accessed January 10, 2016.

19. Pew Internet and American Life Project [webpage on the Internet]. Cell Phones and American Adults. 2010. Available from: http://www.pewinternet.org/2010/09/02/cell-phones-and-american-adults/. Accessed January 10, 2016.

20. Head KJ, Noar SM, Iannarino NT, Harrington NG. Efficacy of text messaging-based interventions for health promotion: a meta-analysis. Soc Sci Med. 2013;97:41-48.

21. Hall AK, Cole-Lewis H, Bernhardt JM. Mobile text messaging for health: a systematic review of reviews. Annu Rev Public Health. 2015;36:393-415.

22. Keoleian V, Polcin D, Galloway GP. Text messaging for addiction: a review. J Psychoactive Drugs. 2015;47(2):158-176.

23. Boulos MN, Brewer AC, Karimkhani C, Buller DB, Dellavalee RP. Mobile medical and health apps: state of the art, concerns, regulatory control and certification. Online J Public Health Inform. 2014;5(3):229-262.

24. Abroms LC, Lee Westmaas J, Bontemps-Jones J, Ramani R, Mellerson J. A content analysis of popular smartphone apps for smoking cessation. Am J Prev Med. 2013;45(6):732-736.

25. Abroms LC, Johnson PR, Heminger CL, et al. Quit4baby: results from a pilot test of a mobile smoking cessation program for pregnant women. JMIR Mhealth Uhealth. 2015;3(1):e10.

26. Naughton F, Prevost AT, Gilbert H, Sutton S. Randomized controlled trial evaluation of a tailored leaflet and SMS text message self-help intervention for pregnant smokers (MiQuit). Nicotine Tob Res. 2012; 14(5):569-577.

27. Naughton F, Jamison J, Sutton S. Attitudes towards SMS text message smoking cessation support: a qualitative study of pregnant smokers. Health Educ Res. 2013;28(5):911-922. doi: 10.1093/her/cyt057.

28. Cooper S, Foster K, Naughton F, et al. Pilot study to evaluate a tailored text message intervention for pregnant smokers (MiQuit): study protocol for a randomised controlled trial. Trials. 2015;16:29.

29. van Mierlo T, Fournier R, Jean-Charles A, Hovington J, Ethier I, Selby P. I'll txt $\mathrm{u}$ if $\mathrm{i}$ have a problem: how the Société Canadienne du cancer in Quebec applied behavior-change theory, data mining and agile software development to help young adults quit smoking. PLoS One. 2014;9(3):e91832.

30. Pollack KI, Lyna P, Bilheimer A, et al. A pilot study testing SMS text delivered scheduled gradual reduction to pregnant smokers. Nicotine Tob Res. 2013;15(10):1773-1776.

31. Bandura A. Social cognitive theory: an agentic perspective. Annu Rev Psychol. 2001;52:1-26.

32. Rosenstock IM, Strecher VJ, Becker MH. Social learning theory and the health belief model. Health Educ Q. 1988;15(2):175-183.

33. Prochaska JO, DiClemente CC. Stages of change in the modification of problem behaviors. Prog Behav Modif. 1992;28:183-218.

34. Roberts NJ, Kerr SM, Smith SMS. Behavioral interventions associated with smoking cessation in the treatment of tobacco use. Health Serv Insights. 2013;6:79-85.

35. Borland R, Balmford J. Perspectives on relapse prevention: an exploratory study. Psychol Health. 2005;20(5):661-671.

36. Petty RE, Cacioppo JT. The Elaboration Likelihood Model of Persuasion. New York: Academic Press; 1986.

37. Abroms LC, Boal AL, Simmens SJ, Mendel JA, Windsor RA. A randomized trial of Text2Quit. A text messaging program for smoking cessation. Am J Prev Med. 2014;47(3):242-250. 


\section{Publish your work in this journal}

Substance Abuse and Rehabilitation is an international, peer-reviewed, open access journal publishing original research, case reports, editorials, reviews and commentaries on all areas of addiction and substance abuse and options for treatment and rehabilitation. The manuscript management system is completely online and includes a very quick and fair peer-review system. Visit http://www.dovepress.com/testimonials.php to read real quotes from published authors. 